Simultaneous electrochemical detection of tramadol and

O-desmethyltramadol with Nafion-coated tetrahedral amorphous carbon electrode

Mynttinen, Elsi

2019-02-01

Mynttinen, E , Wester , N , Lilius , T, Kalso , E , Koskinen , J \& Laurila , T 2019 , '

Simultaneous electrochemical detection of tramadol and O-desmethyltramadol with

Nafion-coated tetrahedral amorphous carbon electrode ', Electrochimica Acta, vol. 295 , pp.

347-353 . https://doi.org/10.1016/j.electacta.2018.10.148

http://hdl.handle.net/10138/309283

https://doi.org/10.1016/j.electacta.2018.10.148

unspecified

publishedVersion

Downloaded from Helda, University of Helsinki institutional repository.

This is an electronic reprint of the original article.

This reprint may differ from the original in pagination and typographic detail.

Please cite the original version. 


\title{
Simultaneous electrochemical detection of tramadol and O-desmethyltramadol with Nafion-coated tetrahedral amorphous carbon electrode
}

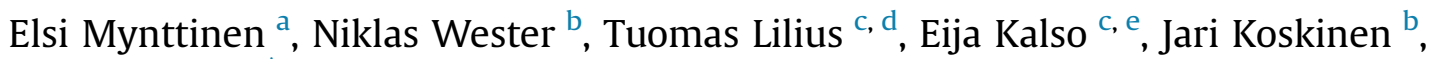 \\ Tomi Laurila ${ }^{\text {a, * }}$ \\ a Department of Electrical Engineering and Automation, Aalto University, Tietotie 3, 02150, Espoo, Finland \\ ${ }^{\mathrm{b}}$ Department of Chemistry and Materials Science, Aalto University, Kemistintie 1, 02150, Espoo, Finland \\ ${ }^{c}$ Department of Pharmacology, University of Helsinki, Haartmaninkatu 8, 00290, Helsinki, Finland \\ ${ }^{\mathrm{d}}$ Department of Clinical Pharmacology, University of Helsinki and Helsinki University Hospital, Tukholmankatu 8C, 00290, Helsinki, Finland \\ e Pain Clinic, Department of Anesthesiology, Intensive Care and Pain Medicine, University of Helsinki and Helsinki University Hospital, Haartmaninkatu 2A, \\ 00290, Helsinki, Finland
}

\section{A R T I C L E I N F O}

\section{Article history:}

Received 31 July 2018

Received in revised form

12 October 2018

Accepted 23 October 2018

Available online 24 October 2018

\section{Keywords:}

Tetrahedral amorphous carbon

Nafion

Tramadol

O-desmethyltramadol

Electrochemical sensor

\begin{abstract}
A B S T R A C T
Tramadol (TR) is a member of the opioid family and is widely used for pain treatment in clinical patient care. The analgesic effect of tramadol is induced primarily by its main metabolite O-desmethyltramadol (ODMT). Due to inter-individual differences in the TR metabolism to ODMT, the responses to TR vary highly between patients. Thus, a fast and selective method for simultaneous detection of TR and ODMT would increase the patient safety and pain treatment efficacy. In this study, a tetrahedral amorphous carbon (ta-C) electrode coated with a thin dip-coated recast Nafion membrane was fabricated for selective electrochemical determination of TR and ODMT. With this Nafion/ta-C electrode, simultaneous detection of TR and ODMT was achieved with linear ranges of $1-12.5 \mu \mathrm{M}$ and $1-15 \mu \mathrm{M}$, respectively. The limits of detection were $131 \mathrm{nM}$ for TR and $209 \mathrm{nM}$ for ODMT. Both analytes were also measured in the presence of several common interferents, demonstrating the high selectivity of the fabricated electrode. In addition, the effect of $\mathrm{pH}$ on the peak potential was studied to observe the electrochemical behavior of the analytes at the electrode. Finally, clinically relevant concentrations of TR and ODMT were simultaneously detected from diluted human plasma to assess the applicability of the electrode in real samples. The fabricated Nafion/ta-C electrode was found successful in the simultaneous electrochemical detection of TR and ODMT in both buffer solution and in human plasma.
\end{abstract}

๑) 2018 Elsevier Ltd. All rights reserved.

\section{Introduction}

Opioids are a group of analgesics widely used for treatment of moderate and severe pain. Tramadol (TR), a prodrug classified as a weak opioid, is frequently used for acute pain relief, especially in postoperative care. The acute analgesic opioid effects of TR are mediated through its main metabolite O-desmethyltramadol (ODMT) (Fig. 1) [1]. TR itself has no opioid activity but has antinociceptive effects through the inhibition of norepinephrine

\footnotetext{
* Corresponding author.

E-mail addresses: elsi.mynttinen@aalto.fi (E. Mynttinen), niklas.wester@aalto.fi (N. Wester), tuomas.lilius@helsinki.fi (T. Lilius), eija.kalso@helsinki.fi (E. Kalso), jari.koskinen@aalto.fi (J. Koskinen), tomi.laurila@aalto.fi (T. Laurila).
}

reuptake [2,3], making it effective also in the treatment of neuropathic pain.

The metabolism from TR into ODMT is mediated through the hepatic cytochrome P450 (CYP) 2D6 enzyme. Due to polymorphisms within the CYP2D6 gene, the activity of the CYP2D6 enzyme can vary, making metabolic rates of TR into ODMT highly individual. Several commonly used drugs also inhibit CYP2D6 activity, further altering the TR-ODMT metabolism. Consequently, the responses to a given dose of TR can range from no effect to serious adverse effects by overdose, making the correct dosing of TR extremely challenging in clinical patient care.

Meanwhile, the currently available methods for determining opioid concentrations in patient samples are standard analytical tools, including high-performance liquid chromatography and 


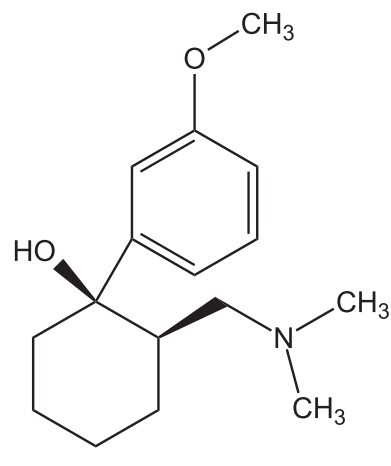

Tramadol

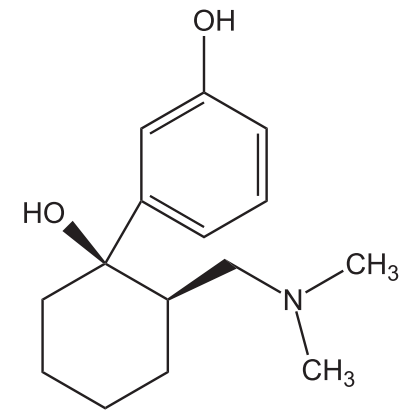

O-desmethyltramadol
Fig. 1. The chemical structures of tramadol and O-desmethyltramadol.

liquid chromatography coupled with mass spectrometry [4]. Although highly accurate, these methods suffer from complex protocols with time-consuming processes that require high level of expertise to conduct. Consequently, opioid blood concentrations are not routinely measured in clinical patient care, mostly due to the lack of affordable and fast methods. Thus, the current administration of opioids is based on standard dosages, careful titration of the dose, and the experience of the medical personnel. Therefore, there is a great need for a fast and accurate method for determining opioid concentrations.

Carbon-based electrochemical sensors have been extensively studied for the detection of biomolecules due to their high sensitivity, affordability and fast response times [5]. Tetrahedral amorphous carbon (ta-C) is a form of diamond-like carbon (DLC) with $\mathrm{sp}^{3}$ fraction above 60\% [6]. It is resistant to wear, corrosion [7] and biofouling [8-12] and has a wide water window and low background current $[13,14]$. Unlike boron doped diamond (BDD), it can be deposited and patterned with room temperature processes. Moreover, the physicochemical properties of ta- $\mathrm{C}$ can be tuned by doping with nitrogen as well as by controlling the $\mathrm{sp}^{2} / \mathrm{sp}^{3}$ ratio [15-18].

With undoped ta-C films, the overall electron transfer kinetics can be controlled by varying the thickness of the film. In previous studies, we have found that ta-C films with thicknesses in the range of $7-15 \mathrm{~nm}$ support facile electron transfer [13,14]. For thicker films, the electron transport through the film slows down the overall oxidation kinetics, whereas thinner films contain pinholes, leading to the substrate material dominating the electrochemical properties. Due to its favorable properties, ultrathin ta-C films have been used for enzymatic hydrogen peroxide sensing [9], detection of drug molecules [19] and neurotransmitters [20,21].

TR has been studied with electrochemical methods in various reports both in buffer and real samples [22-31]. However, since ODMT is the metabolite primarily responsible for the analgesic effect of TR, it would be very important to be able to measure both TR and ODMT. Yet, to our knowledge, there are no reports of simultaneous electrochemical determination of TR and ODMT concentrations.

In biological samples, probably the greatest challenge for detection of TR and ODMT is the presence of large amounts of interferents, such as ascorbic acid (AA) and uric acid (UA). The concentrations of these molecules are in the range of hundreds of micromoles per liter $(100-500 \mu \mathrm{M})$, while the therapeutic concentration of TR is only a couple micromoles per liter, at most [32]. One approach to increase the selectivity of the sensor is to coat it with a polymer membrane. The most commonly used polymer in electrochemical sensors is Nafion, a sulfonated fluoropolymer with negatively charged surface groups. Due to its unique structure, this polymer acts as a permselective membrane, blocking out interfering anionic molecules while allowing cationic analytes to pass through onto the electrode surface and to be detected [33-35]. While both AA and UA are in anionic forms in neutral $\mathrm{pH}$, the majority of opioids, including TR and ODMT, exist mainly as cations. Thus, Nafion can be effectively used to enhance the selectivity of the sensor.

In this work, a carbon-based electrochemical sensor was developed enabling, for the first time, fast and selective simultaneous detection of both TR and ODMT in the presence of various interferents in buffer solution. The electrode was prepared from a tetrahedral amorphous carbon thin film deposited on top of a conductive silicon wafer and coated with a thin dip-coated polymer membrane of Nafion. Differential pulse voltammetry (DPV), a variation of square wave voltammetry (SWV), was selected as the electrochemical detection method. In DPV, the current is recorded at the end of each potential step, minimizing the effect of the nonfaradaic background current and thus increasing the signal-tonoise-ratio. In addition, the response time of DPV is only a couple minutes, a time range well below of that of the currently used methods for opioid concentration determination. DPV was used to measure TR and ODMT concentrations from a buffer solution as well as from a solution in the presence of various interferents, including AA and UA. Further, the applicability of the Nafion-coated electrode in real samples was verified by measuring the analytes simultaneously from diluted human plasma samples. This study provides, for the first time, a simple electrode design using a novel combination of carbon thin film and Nafion membrane for simultaneous detection of TR and ODMT.

\section{Experimental}

\subsection{Preparation of Nafion/ta-C}

The $15 \mathrm{~nm}$ ta-C films were deposited on p-type (100) Si wafers (Siegert) with $<0.005 \Omega \mathrm{cm}$ resistivity. The films were deposited directly on the Si wafers without adhesion layers by means of filtered cathodic vacuum arc (FCVA). The deposition process is described in detail in Ref. [14]. The physical and electrochemical properties of these films have been studied in great detail in previous work [13,14,18-20].

The prepared wafer was diced into squares with sides of $1 \mathrm{~cm}$. The sample was placed on a piece of conductive copper clad (double sided FR4 fiber glass board, Elprintta, Finland) and the contact between the sample and the copper support was enhanced by scraping the Si-side with a diamond scriber and a piece of copper. The electrode was then enclosed in a polytetrafluoroethylene film (PTFE, Saint-Gobain Performance Plastics CHR 22552) with a $6 \mathrm{~mm}$ (or $3 \mathrm{~mm}$ ) hole to define the electrode area and to isolate the copper sheet from the electrolyte solution. Before Nafion coating, a $2.5 \mathrm{wt}-\%$ Nafion solution was prepared by diluting $5 \mathrm{wt}-\%$ Nafion 117 solution (Sigma Aldrich) in 94 wt-\% ethanol. Finally, the electrode was immersed in the $2.5 \mathrm{wt}$-\% Nafion solution for $5 \mathrm{~s}$ and allowed to dry under ambient conditions overnight.

\subsection{Characterization of Nafion/ta-C electrodes}

The suitable concentration of the Nafion solution for coating was determined by preparing coatings with several Nafion concentrations and testing their filtering ability as well as the capability of detecting TR and ODMT. ta-C electrodes were coated with $0.1,0.5,1,2.5$ and $5 \%$ Nafion solutions by immersion for $5 \mathrm{~s}$, and each electrode measured in $500 \mu \mathrm{M}$ ascorbic acid (Sigma-Aldrich) and $500 \mu \mathrm{M}$ uric acid (Sigma-Aldrich) in $0.01 \mathrm{M}$ phosphate buffered 
saline (PBS, pH 7.4) with DPV. Further, the electrodes with $2.5 \%$ and $5 \%$ Nafion coating were also measured in $50 \mu \mathrm{M}$ TR and ODMT.

The thickness of the Nafion membrane was determined with cross-sectional SEM-imaging by a Hitachi S-4700 SEM at $15 \mathrm{kV}$ accelerating voltage. Cross-sectional SEM samples were prepared along the $6 \mathrm{~mm}$ diameter of the circular electrodes by wafer cleaving techniques in liquid nitrogen. To obtain an average film thickness, images with magnifications ranging from $5 \mathrm{k}$ to $200 \mathrm{k}$ were taken in intervals of about $100 \mu \mathrm{m}$ across the cross section and the thickness of the film in each image was calculated as an average of three points. The water window of the Nafion/ta-C electrode was established by a cyclic voltammetry (CV) measurement in PBS with a scan rate of $500 \mathrm{mV} / \mathrm{s}$.

\subsection{Electrochemical measurements}

DPV measurements were performed using a $\mathrm{CH}$ Instruments (CHI630E) potentiostat. A three-electrode cell was used for all electrochemical measurements with an $\mathrm{Ag} / \mathrm{AgCl}$ electrode as reference $(+0.199 \mathrm{~V}$ vs standard hydrogen electrode, Radiometer Analytical) and a platinum wire as the counter electrode. In all measurements, the pulse amplitude was $50 \mathrm{mV}$, pulse width $0.05 \mathrm{~s}$ and pulse period $0.2 \mathrm{~s}$. All solutions (except plasma) were deoxygenated with $\mathrm{N}_{2}$ for at least 5 minutes before the measurements and the cell was kept at $\mathrm{N}_{2}$ overpressure during the measurements.

The Nafion/ta-C electrodes were always measured the following day after coating and were allowed to swell in PBS for 30 min prior to starting the experiments. Background scans were measured for all electrodes at least ten times or until the background current was stable. For determination of the limits of detection, three additional subsequent backgrounds were recorded for each electrode. The electrodes were always kept in PBS between each measurement to prevent the Nafion film from drying.

The DPV scans in PBS buffer solution were measured for 1, 10, 25, and $50 \mu \mathrm{M}$ of tramadol hydrochloride $\left(\right.$ Tramal $^{\circledR} 50 \mathrm{mg} / \mathrm{mL}$, Orion Pharma, Finland) and O-desmethyltramadol hydrochloride (SigmaAldrich) in separate solutions, by successively injecting the analytes into the cell with a pipette from a $1 \mathrm{mM}$ stock solution. The measurements for TR were done in a potential window of $-0.4 \mathrm{~V}$ to $1.8 \mathrm{~V}$ and for ODMT from $-0.4 \mathrm{~V}$ to $1.6 \mathrm{~V}$. The DPVs were measured with both plain and Nafion-coated ta-C electrodes ( $6 \mathrm{~mm}$ diameter) to study the effects of the Nafion film on the signals for TR and ODMT.

For the concentration series of TR and ODMT, DPVs were measured in PBS containing either $10 \mu \mathrm{M}$ of TR or $5 \mu \mathrm{M}$ ODMT. TR or ODMT were then successively added from $1 \mathrm{mM}$ stock solutions with a pipette into the cell to obtain concentrations of $1,2.5,5,7.5$, $10,12.5$ and $15 \mu \mathrm{M}$ and measured with a Nafion-coated ta-C electrode ( $6 \mathrm{~mm}$ diameter). In these measurements, the electrodes were kept in PBS for 5 min between each concentration. The average peak current densities of three electrodes were plotted against the analyte concentration for both TR and ODMT and the linear ranges obtained. The values for the limit of detection (LOD) were calculated by the formula $\operatorname{LOD}=3.3 \times \sigma / s$, where $\sigma$ is the standard deviation of three successive background currents in $\mu \mathrm{A}$ (at the oxidation potential of the analyte) and $s$ is the sensitivity of the electrode $(\mu \mathrm{A} / \mu \mathrm{M})$. The values were calculated as the average of three electrodes.

The effects of $\mathrm{pH}$ on the peak potentials were studied in $0.01 \mathrm{M}$ phosphate buffer (PB) solutions with different pH-values: 3 $(3.014 \pm 0.005), \quad 5 \quad(4.977 \pm 0.005), \quad 7 \quad(7.062 \pm 0.005), \quad 9$ $(8.980 \pm 0.005)$ and $11(11.000 \pm 0.005)$. DPVs were measured for $25 \mu \mathrm{M}$ TR and $25 \mu \mathrm{M}$ ODMT in separate solutions with a plain ta-C electrode ( $3 \mathrm{~mm}$ diameter). Interference studies were done with biologically relevant concentrations of several common interferents: $250 \mu \mathrm{M}$ and $500 \mu \mathrm{M}$ AA and UA, $250 \mu \mathrm{M}$ ibuprofen (IBU, Sigma-Aldrich), $500 \mu \mathrm{M}$ acetaminophen (Sigma-Aldrich), $5 \mu \mathrm{M}$ diclofenac (Sigma-Aldrich), $150 \mu \mathrm{M}$ citric acid (Sigma-Aldrich) and $5 \mathrm{mM}$ glucose (Sigma-Aldrich). The interferents were added into a PBS solution with $10 \mu \mathrm{M}$ TR and $5 \mu \mathrm{M}$ ODMT and measured separately with a Nafion/ta-C electrode.

For the plasma measurements, expired human plasma (Octaplas $A B$, Sweden) was received from the blood center of HUSLAB (Finland). The plasma was diluted as 1:10 in PBS, and equal additions of TR and ODMT were injected from $1 \mathrm{mM}$ stock solutions to generate concentrations of $5,10,15$ and $20 \mu \mathrm{M}$ of both analytes. DPVs were measured from each concentration with a ta-C/Nafion electrode $(6 \mathrm{~mm}$ diameter), keeping the electrodes in PBS for 5 minutes between each addition. The concentration current density curves were plotted for both analytes with standard deviations, with $n=3$. Other experiment settings were as above.

\section{Results and discussion}

\subsection{Characterization of the Nafion/ta-C electrode}

The ta-C thin films have been thoroughly characterized in previous work [13,14,18-20]. On the contrary, the behavior of thin Nafion layers has not been systematically studied before and is thus an ongoing area of research in our group. The $2.5 \%$ Nafion concentration for the coating was selected based on its ability to sufficiently filter out major interferents while still presenting well defined oxidation peaks for TR and ODMT (Fig. S1). The characterization by SEM-imaging showed that the immersion of the electrode in $2.5 \%$ Nafion solution produced a film with an average thickness of $460 \mathrm{~nm}$ with a standard deviation of $640 \mathrm{~nm}$ (Fig. S2). It should be noted that, due to the coating method, the Nafion film thickness was somewhat higher in close proximity to the edge of the PTFE film (excluded in the average thickness). The water window of this hybrid electrode was found to be approximately $5 \mathrm{~V}$ in PBS (Fig. S3), which is very close to that of bare ta-C [36].

\subsection{Electrochemical measurements in buffer solution}

Differential pulse voltammetry (DPV) was used as the electrochemical method to determine TR and ODMT concentrations in a buffer solution. Several concentrations, 1, 10, 25 and $50 \mu \mathrm{M}$, of TR and ODMT were measured in PBS with both plain and Nafion/ta-C electrodes. Fig. 2 shows that without Nafion, three oxidation peaks for TR and two for ODMT are seen on plain ta-C, while with the Nafion-coating, only one well-defined peak is detected for each molecule. In addition, the Nafion-coated electrodes exhibit better linearity for the peak current density as a function of analyte concentration than that of the plain ta-C (insets in Fig. 2B and D, current densities background subtracted). A possible explanation for this could be a change in the reaction kinetics, shifting more towards diffusion control under the Nafion film. A better linearity is observed especially at the lower concentration range which, indeed, is the more relevant range considering clinical applications.

For TR, a probable oxidation mechanism involving the amine group has been provided [24,37], consisting of two separate electron transfers, a proton transfer between the two and a subsequent chemical reaction. On the ta-C electrode, the first peaks at $1.1 \mathrm{~V}$ and 1.4 (at pH 7.4) could be attributed to oxidation of the amine group. However, no definite conclusions can be drawn based on these experiments alone. Furthermore, the third peak has not been observed at neutral $\mathrm{pH}$ in any other study found in the literature (see Table 1), and cannot be explained by previous findings. The emergence of this additional peak at the higher potential of $1.7 \mathrm{~V}$ (without Nafion) is only enabled by the exceptionally large water 


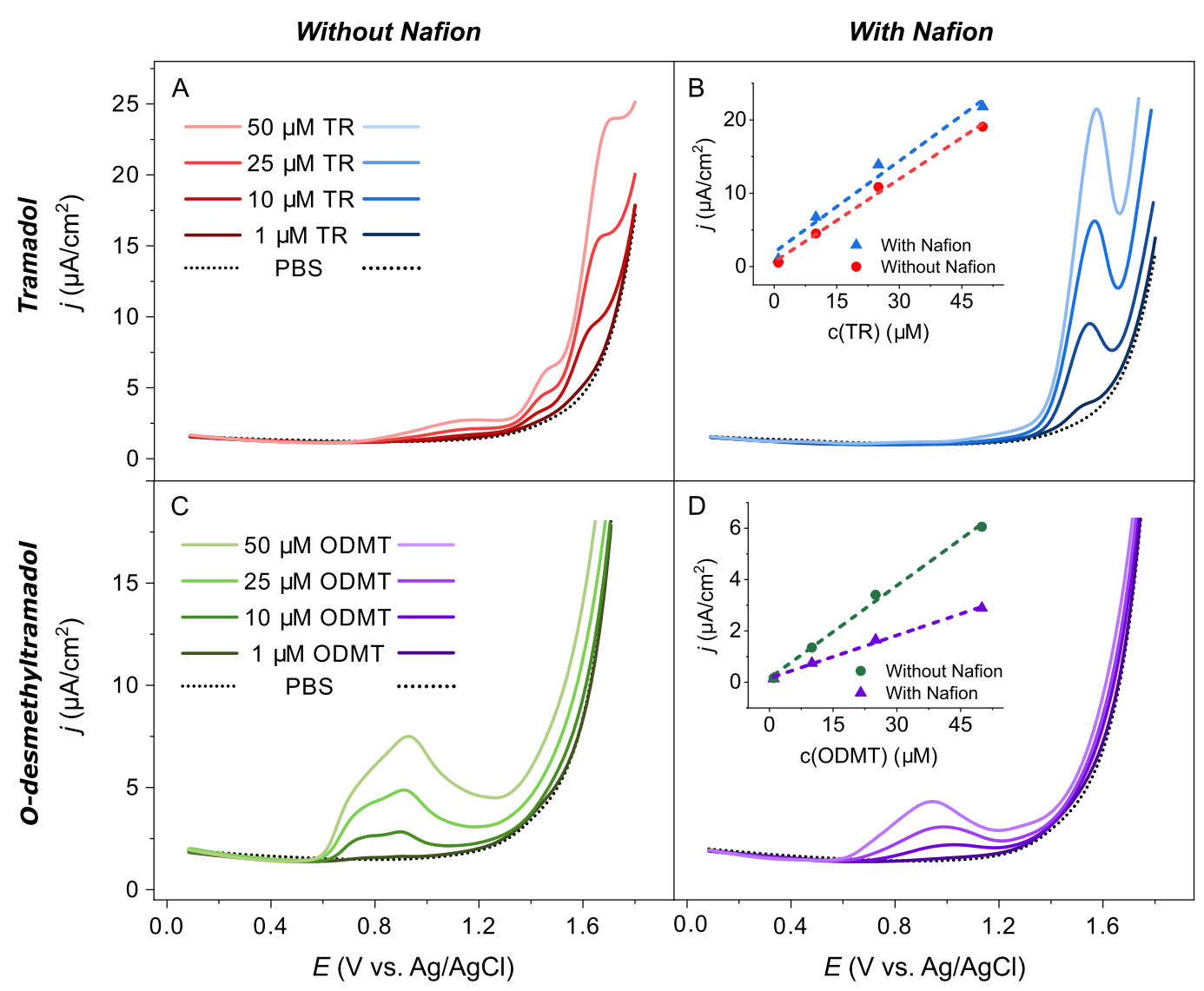

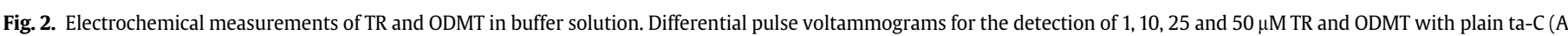

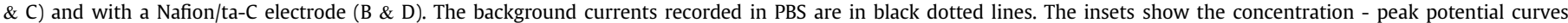
(background subtracted) for coated and uncoated electrodes.

Table 1

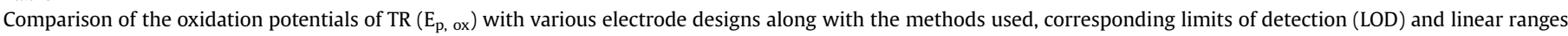
obtained.

\begin{tabular}{|c|c|c|c|c|c|}
\hline Electrode & Method & $\mathrm{E}_{\mathrm{p}, \text { ox }}(\mathrm{V})$ & $\mathrm{LOD}(\mu \mathrm{mol} / \mathrm{L})$ & Linear range $(\mu \mathrm{mol} / \mathrm{L})$ & Ref. \\
\hline MWCNTs/GCE & DPV & 0.55 & 0.361 & $2-300$ & [23] \\
\hline $\mathrm{CNP} / \mathrm{GCE}$ & DPV & 0.6 & 1.0 & $10-1000$ & [22] \\
\hline LDH/Fe304@GCE & DPV & 0.7 (pH 9.5) & 0.3 & $1-200$ & [30] \\
\hline D50wx2-GNP-GCPE & AdSSWV & $0.7(\mathrm{pH} \mathrm{6})$ & 0.011 & $0.033-42.2$ & [24] \\
\hline GO-MWCNTs/CPE & DPV & 0.75 & 0.00015 & $0.002-1100$ & [31] \\
\hline GPE & DPV & $0.75(\mathrm{pH} 9.2)$ & 0.0038 & $0.1-1.5$ & [29] \\
\hline MIP-MWCNT/CPE & SWV & 0.8 & 0.004 & $0.01-20$ & [25] \\
\hline NiFe2O4NPs/Gr/CPE & SWV & $0.8(\mathrm{pH} 9.5)$ & 0.03 & $0.01-9$ & [26] \\
\hline PNBMGCE & DPV & 0.9 & 0.5 & $1-310$ & [28] \\
\hline BDDE & FIA-MPA* & $1.6(\mathrm{pH} 2)$ & 0.04 & $0.08-10$ & [27] \\
\hline \multirow[t]{2}{*}{ Nafion/ta-C } & DPV & 1.5 (TR) & 0.13 & $1-12.5$ & This work \\
\hline & & 0.95 (ODMT) & 0.21 & $1-15$ & \\
\hline
\end{tabular}

MWCNTs/GCE = Glassy carbon electrode modified with multi-walled carbon nanotubes.

$\mathrm{CNP} / \mathrm{GCE}=$ Glassy carbon electrode modified with carbon nanoparticles.

$\mathrm{LDH} / \mathrm{Fe}_{3} \mathrm{O}_{4} @ \mathrm{GCE}=$ Glassy carbon electrode with magneto layer double hydroxide $\mathrm{Fe}_{3} \mathrm{O}_{4}$.

D50wx2-GNP-GCPE = Glassy carbon paste electrode modified with D50wx2 and gold nanoparticles, AdSSWV = Adsorptive stripping SWV

GO-MWCNTs/CPE = Carbon paste electrode modified with graphene oxide and multi-walled carbon nanotubes

$\mathrm{GPE}=$ Graphite pencil electrode.

MIP-MWCNT/CPE = Carbon paste electrode modified with nano-molecularly imprinted polymer and multi-walled carbon nanotubes.

$\mathrm{NiFe}_{2} \mathrm{O}_{4} \mathrm{NPs} / \mathrm{Gr} / \mathrm{CPE}=$ Carbon paste electrode modified with graphite and $\mathrm{NiFe}_{2} \mathrm{O}_{4}$ nanoparticles.

$\mathrm{PNBMGCE}=\mathrm{A}$ poly(Nile blue)modified glassy carbon electrode.

$\mathrm{BDDE}=$ Boron-doped diamond electrode, FIA-MPA = Flow injection analysis with multiple pulse amperometric detection, *Same ox potential also with CV.

window of the ta-C electrode material. It can be speculated that this peak would be a result of further oxidation of the amine group or oxidation of the hydroxyl group of the molecule. However, further experiments are required to fully explain this behavior.
The electrochemical oxidation reactions of ODMT have not, to our knowledge, been studied in the literature before. The in depth analysis of such mechanisms will necessarily require extensive amount of additional experiments and is therefore outside the 
scope of this study. Similarly, the disappearance of the additional peaks for TR and ODMT could be explained by the different behavior of the functional groups of the analytes at the electrodeNafion interface. However, analyzing the specific mechanisms of these interactions will be a topic of future research.

From the initial DPV measurements in buffer solution, it can be seen that the Nafion coating enables a simultaneous detection of TR and ODMT on the ta-C electrode. Fig. 3 shows DPV measurements for several concentrations of TR in the presence of ODMT (Fig. 3A) and vice versa (Fig. 3B) in buffer solution. The insets show the linear detection range for both analytes (current densities background subtracted) with error bars displaying the standard deviation between electrodes $(n=3)$. A linear range for TR was obtained in a concentration range of $1-12.5 \mu \mathrm{M} \quad\left(j=0.3994 c_{\mathrm{TR}}-0.0452\right.$, $\left.r^{2}=0.992\right)$ and $1-15 \mu \mathrm{M}$ for ODMT $\left(j=0.0571 c_{\text {ODMT }}-0.0086\right.$, $\left.r^{2}=0.998\right)$. The values for limit of detection were $131 \mathrm{nM}$ for TR and $209 \mathrm{nM}$ for ODMT. Although not yet optimal, these limits are quite
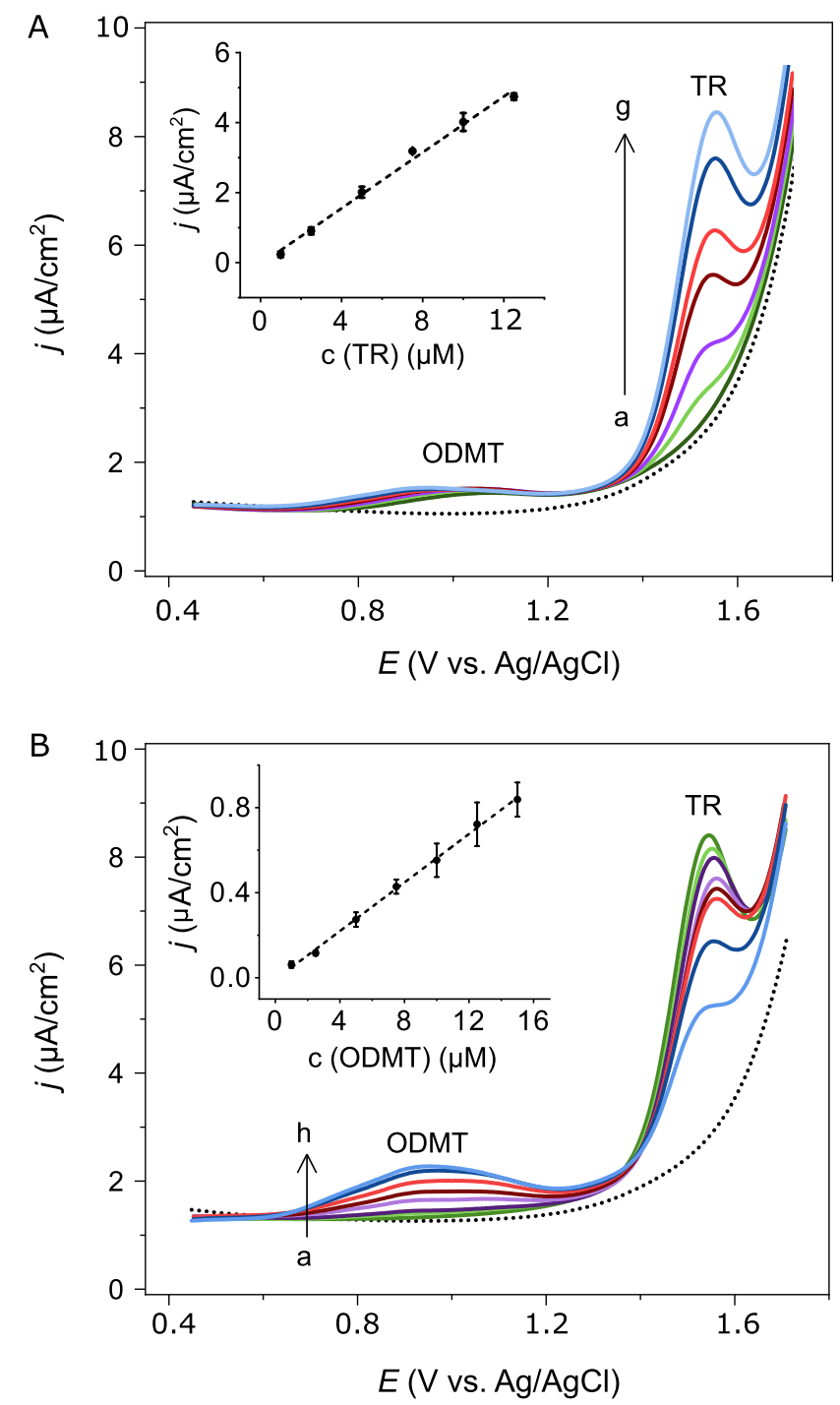

Fig. 3. Electrochemical measurements with concentration series of TR and ODMT in buffer solution. Differential pulse voltammograms of (A) $5 \mu \mathrm{M}$ ODMT and $0,1,2.5,5$, $7.5,10$ and $12.5 \mathrm{TR}(\mathrm{a}-\mathrm{g})$ and $(\mathrm{B}) 10 \mu \mathrm{M}$ TR and $0,1,2.5,5,7.5,10,12.5$ and $15 \mu \mathrm{M}$ ODMT $(\mathrm{a}-\mathrm{h})$. Black dotted line is the background in PBS without analytes. Insets show the linear range for TR and ODMT average peak currents (background subtracted) with standard deviations, $n=3$. A Nafion/ta-C was used as the electrode. sufficient, the clinically relevant concentrations being $0.2-4.2 \mu \mathrm{M}$ for TR and 88-230 nM for ODMT [32].

In Fig. 3B, a significant decrease of the TR peak current is observed as the concentration of ODMT is increased. However, this attenuation of the oxidation current was also seen when multiple scans were measured sequentially in the absence of ODMT with a single electrode. It was found that when TR was measured several times in a row separately without ODMT, the current density was decreased by approximately $20 \%$ by the 5 th DPV cycle (Fig. S6B), as is the case in Fig. 3B. This demonstrated that it is not the addition of ODMT but rather the repetitive use of a single electrode that is responsible for the passivation of the electrode and decrease of the TR peak current. The general passivation behavior could be attributed to the characteristics of the Nafion film, for example partial binding of TR molecules to the sulfonic groups of Nafion. Nevertheless, if the sensor is applied as a disposable test strip, these findings do not pose practical limitations.

\subsection{Effect of $p H$}

The effect of $\mathrm{pH}$ on the electrochemical behavior of TR and ODMT was studied to obtain further information about the electrochemical behavior of the analytes on ta-C (Fig. S7). With TR, the first oxidation peak, as discussed in Section 3.2, is likely due to the two electron transfers of the suggested oxidation mechanism of TR [24]. This would also explain the shift of the oxidation potential to less positive values as the $\mathrm{pH}$ increases, as well as the significant decrease of this peak current at $\mathrm{pH}$ below 7, a behavior also observed in previous studies [24,25]. The peak potential follows a linear equation $E_{\mathrm{p} \text {, ox }}(\mathrm{mV})=-31.7 \mathrm{pH}+1095, r^{2}=0.954$, suggesting the involvement of an unequal number of protons and electrons. The second peak only appears at $\mathrm{pH} 7$ and below, and its peak potential is virtually independent on $\mathrm{pH}$. The third peak does not shift considerably with $\mathrm{pH}$, but completely disappears at $\mathrm{pH} 3$.

The first oxidation peak of ODMT at $0.55 \mathrm{~V}$ (at $\mathrm{pH} 11$ ) follows the linear equation $E_{\mathrm{p} \text {, ox }}(\mathrm{mV})=-46.9 \mathrm{pH}+1069, r^{2}=0.990$, suggesting roughly an equal number of protons and electrons in this reaction. Since this peak does not appear for TR, it can be speculated that it is due to oxidation of the hydroxyl group of ODMT. From these results, it could be proposed that the measurements should be done at $\mathrm{pH} 3$ due to good separation of the peaks for TR and ODMT. However, since the $\mathrm{pK}_{\mathrm{a}}$-values for AA and UA are 4.7 and 5.4, respectively, conducting the measurements in lower $\mathrm{pH}$ would essentially decrease the fraction of negatively charged AA and UA in the solution. This, in turn, would reduce their filtration by the Nafion membrane and could increase the interference significantly. Determining whether altering the $\mathrm{pH}$ would eventually be beneficial for the end application, will require further detailed studies.

\subsection{Interference studies}

Some of the most common interfering molecules present in real samples were measured in buffer solution simultaneously with $5 \mu \mathrm{M}$ ODMT and $10 \mu \mathrm{MTR}$. From these molecules, ibuprofen, diclofenac, citric acid and glucose caused almost no interference with the peak current of TR or ODMT (Fig. S5). The error percentages were between 0.4 and $14.3 \%$ for TR and $0.9-5.6 \%$ for ODMT peak current. This was quite expected behavior, since these interferents are either electrochemically inactive (i.e. glucose) or negatively charged at $\mathrm{pH} 7$.

Separate signals for TR and ODMT could be obtained also in the presence of $250 \mu \mathrm{M}$ and $500 \mu \mathrm{M}$ AA and UA (Fig. S5). Unfortunately, the interference from these molecules was somewhat higher, the error percentages being between 3.6 and $15.6 \%$ for TR and 17.5-31.0\% for ODMT. However, the error caused by AA and UA was 
not consistent with the amount of the interferents, but had a rather wide deviation. Since this behavior is most probably due to the properties of the Nafion layer, it will be possible to further optimize the performance of the electrode by modifying the features of this polymer membrane.

\subsection{Electrochemical measurements in plasma}

Electrochemical measurements were conducted in human plasma, diluted ten times in PBS buffer. Fig. 4 shows the background currents first in PBS with Nafion/ta-C electrode and then in plasma with plain and Nafion/ta-C electrode, demonstrating the necessity of using Nafion when applying the sensor in real samples. While the background current increases somewhat in plasma compared to PBS when measured with Nafion/ta-C, it is evident that any possible signals, especially from ODMT, would be buried under the interference without the Nafion filter.

Fig. 5 presents the results from DPV measurements for both TR and ODMT, simultaneously added into the diluted plasma solution. Concentrations of $5,10,15$ and $20 \mu \mathrm{M}$ of both analytes were measured with a Nafion/ta-C electrode. The inset shows the average peak current densities (background subtracted) with error bars corresponding to the standard deviations between three electrodes. A linear response between 5 and $15 \mu \mathrm{M}$ was obtained for TR with $j=0.2466 c_{\mathrm{TR}}-0.0553, r^{2}=0.910$ and for ODMT with $j=0.0646 c_{\text {ODMT }}-0.0904, r^{2}=0.970$. The slopes of the analyte current responses differ slightly compared to those obtained from the buffer solution due to the matrix effect presented by the plasma. The linearity of the concentration - current density curves is also broken after $15 \mu \mathrm{M}$ in both cases, the effect being considerably stronger for TR than ODMT. This indicates some degree of passivation of the electrode surface, a phenomenon probably amplified by the plasma proteins. A major contribution is also likely due to repeated measurements with a single electrode and the saturation of the channel structure of Nafion, as discussed in section 3.2. However, as mentioned before, this effect would not be a major concern when designing a disposable measurement strip.

The presented hybrid Nafion/ta-C electrode was able to electrochemically detect TR and ODMT in both buffer solution and human plasma. Compared to the work from the literature exhibited

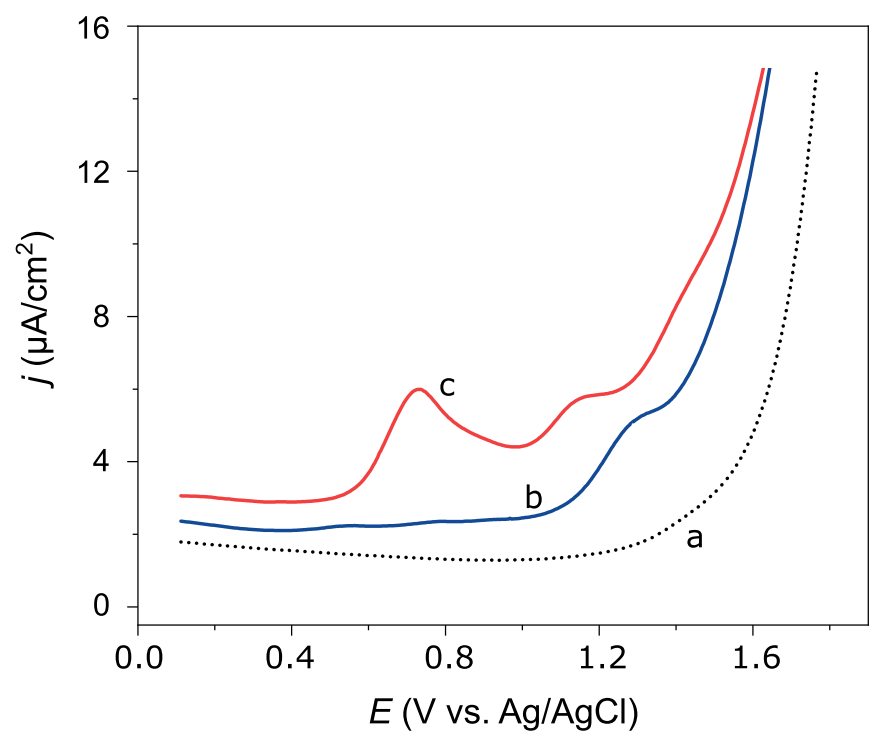

Fig. 4. DPV recordings of background currents in PBS with Nafion-coated ta-C electrode (a) and in diluted plasma (1:10 in PBS) with Nafion/ta-C (b) and bare ta-C (c).

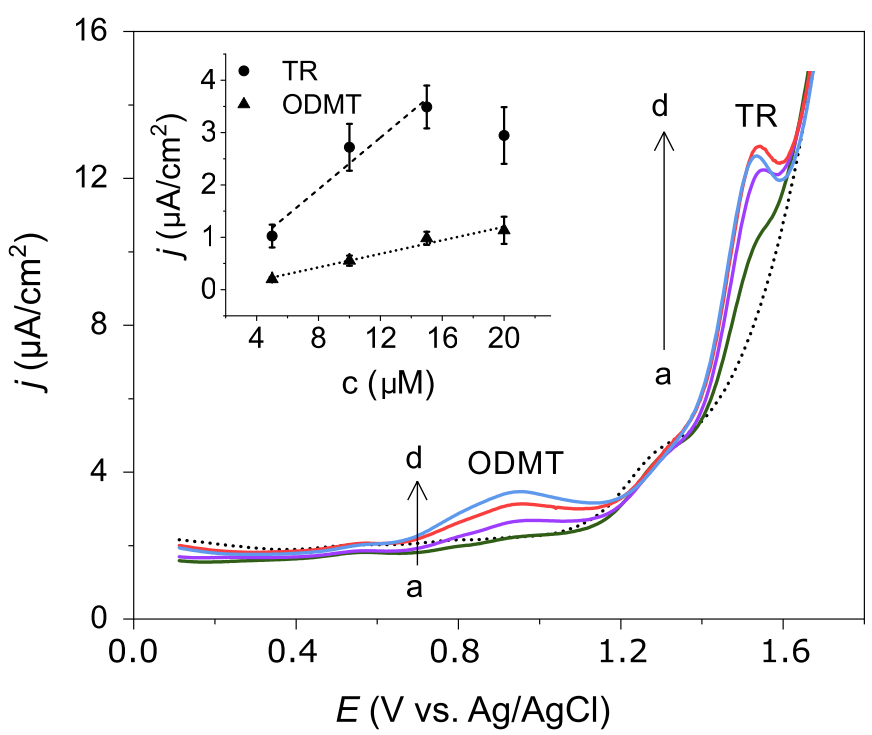

Fig. 5. Simultaneous electrochemical measurements of TR and ODMT in human plasma. Differential pulse voltammograms of concentrations of 5, 10, 15 and 20 (a-d) of TR and ODMT in diluted plasma, analytes injected with equal additions. Black dotted line is the background in plasma without analytes. The inset shows the average peak currents as a function of concentration for both TR and ODMT with error bars from standard deviations, $n=3$.

in Table 1, the limit of detection and linear range for TR obtained in this work are adequate and more importantly, clinically relevant. The divergent oxidation potential observed for TR at the Nafion/taC electrode can be completely attributed to the unique properties of this hybrid electrode. This combination of materials enabled the simultaneous measurement of TR and its metabolite ODMT, presenting its potential for further development into a real-time sensor for the detection of these analytes. Since the fabrication of the Nafion/ta-C electrode is simple and is fabricated as a thin film assembly, this electrode has a variety of desirable properties for a fast and affordable method for point-of-care detection of TR, ODMT and other opioids.

\section{Conclusions}

In summary, we showed here that with a Nafion/ta-C hybrid electrode, we were able to simultaneously detect small concentrations of TR and ODMT in both buffer solution and human plasma. With differential pulse voltammetry, linear current responses for TR and ODMT were obtained in the concentration ranges of $1-12.5 \mu \mathrm{M}$ and $1-15 \mu \mathrm{M}$, respectively. In addition, the effect of $\mathrm{pH}$ was studied to further understand the behavior of the analytes at the electrode and in the Nafion film. From the interference studies, it was concluded that the electrode showed sufficient selectivity towards the analytes of interest. Furthermore, there was no considerable interference observed from the plasma matrix, and linear dependences of current density as a function of concentration were obtained from simultaneous additions of TR and ODMT. Thus, this sensor shows great promise for applications in real-time detection of these analytes as well as other drug molecules in clinical settings.

\section{Conflict of interest}

The authors declare no conflict of interest. 


\section{Acknowledgements}

This work was supported by Business Finland (FEDOC project grant number 211637) and the Aalto ELEC Doctoral School. Jarkko Etula and Tom Lindqvist are acknowledged for fabricating the ta-C samples and Jarkko Etula also for conducting the SEM-imaging. Sara Tuominen is acknowledged for helping with some of the interference studies. The authors also thank Jenni Viinamäki for providing the plasma samples for this work.

\section{Appendix A. Supplementary data}

Supplementary data to this article can be found online at https://doi.org/10.1016/j.electacta.2018.10.148.

\section{References}

[1] L. Gong, U.M. Stamer, M.V. Tzvetkov, R.B. Altman, T.E. Klein, PharmGKB summary: tramadol pathway, Pharmacogenetics Genom. 24 (2014) 374-380. https://doi.org/10.1097/FPC.0000000000000057.

[2] R.B. Raffa, E. Friderichs, W. Reimann, R.P. Shank, E.E. Codd, J.L. Vaught, Opioid and nonopioid components independently contribute to the mechanism of action of tramadol, an 'atypical' opioid analgesic, J. Pharmacol. Exp. Ther. 260 (1992) 275. LP-285.

[3] J.A. Desmeules, V. Piguet, L. Collart, P. Dayer, Contribution of monoaminergic modulation to the analgesic effect of tramadol, Br. J. Clin. Pharmacol. 41 (1996) 7-12. https://doi.org/10.1111/j.1365-2125.1996.tb00152.x.

[4] L. Vlase, S.E. Leucuta, S. Imre, Determination of tramadol and O-desmethyltramadol in human plasma by high-performance liquid chromatography with mass spectrometry detection, Talanta 75 (2008) 1104-1109. https://doi.org/ 10.1016/j.talanta.2008.01.006.

[5] T. Laurila, S. Sainio, M. Caro, Hybrid carbon based nanomaterials for electrochemical detection of biomolecules, Prog. Mater. Sci. 88 (2017) 499-594. https://doi.org/10.1016/j.pmatsci.2017.04.012.

[6] C. Casiraghi, J. Robertson, A.C. Ferrari, Diamond-like carbon for data and beer storage, Mater. Today 10 (2007) 44-53. https://doi.org/10.1016/S13697021(06)71791-6.

[7] J. Robertson, Diamond-like amorphous carbon, Mater. Sci. Eng. R Rep. 37 (2002) 129-282.

[8] E. Kaivosoja, S. Sainio, J. Lyytinen, T. Palomäki, T. Laurila, S.I. Kim, J.G. Han, J. Koskinen, Carbon thin films as electrode material in neural sensing, Surf Coatings Technol. 259 (2014) 33-38. https://doi.org/10.1016/j.surfcoat.2014. 07.056.

[9] N. Tujunen, E. Kaivosoja, V. Protopopova, J.J. Valle-Delgado, M. Österberg, J. Koskinen, T. Laurila, Electrochemical detection of hydrogen peroxide on platinum-containing tetrahedral amorphous carbon sensors and evaluation of their biofouling properties, Mater. Sci. Eng. C 55 (2015) 70-78. https://doi.org/ 10.1016/j.msec.2015.05.060.

[10] N. Isoaho, E. Peltola, S. Sainio, N. Wester, V. Protopopova, B.P. Wilson, J. Koskinen, T. Laurila, Carbon nanostructure based platform for enzymatic glutamate biosensors, J. Phys. Chem. C 121 (2017) 4618-4626. https://doi.org/ 10.1021/acs.jpcc.6b10612.

[11] E. Peltola, N. Wester, K.B. Holt, L.-S. Johansson, J. Koskinen, V. Myllymäki, T. Laurila, Nanodiamonds on tetrahedral amorphous carbon significantly enhance dopamine detection and cell viability, Biosens. Bioelectron. 88 (2017) 273-282. https://doi.org/10.1016/j.bios.2016.08.055.

[12] E. Peltola, S. Sainio, K.B. Holt, T. Palomäki, J. Koskinen, T. Laurila, Electrochemical fouling of dopamine and recovery of carbon electrodes, Anal. Chem. 90 (2018) 1408-1416. https://doi.org/10.1021/acs.analchem.7b04793.

[13] V.S. Protopopova, N. Wester, M.A. Caro, P.G. Gabdullin, T. Palomäki, T. Laurila, J. Koskinen, Ultrathin undoped tetrahedral amorphous carbon films: thickness dependence of the electronic structure and implications for their electrochemical behaviour, Phys. Chem. Chem. Phys. 17 (2015) 9020-9031. https:/ doi.org/10.1039/c4cp05855k.

[14] T. Palomäki, N. Wester, M.A. Caro, S. Sainio, V. Protopopova, J. Koskinen, T. Laurila, Electron transport determines the electrochemical properties of tetrahedral amorphous carbon (ta-C) thin films, Electrochim. Acta 225 (2017) 1-10. https://doi.org/10.1016/j.electacta.2016.12.099.

[15] W. Volksen, R.D. Miller, G. Dubois, Low dielectric constant materials, Chem Rev. 110 (2010) 56-110. https://doi.org/10.1021/cr9002819.

[16] L.-C. Cheng, T.-F. Hung, P.-H. Lee, I.-C. Lin, H.-L. Wen, L.-H. Lu, C.-L. Chiu, S.C. Chen, J.C. Sung, B.-J. Weng, R.-S. Liu, Electrochemical reduction of highefficiency ozone generation through nitrogen-doped diamond-like carbon electrodes, RSC Adv. 3 (2013) 5917-5925. https://doi.org/10.1039/ c3ra23335a.

[17] A. Zeng, V.F. Neto, J.J. Gracio, Q.H. Fan, Diamond-like carbon (DLC) films as electrochemical electrodes, Diam. Relat. Mater. 43 (2014) 12-22. https://doi. org/10.1016/j.diamond.2014.01.003.

[18] S. Sainio, D. Nordlund, M.A. Caro, R. Gandhiraman, J. Koehne, N. Wester, J. Koskinen, M. Meyyappan, T. Laurila, Correlation between $\mathrm{sp}^{3}$-to- $\mathrm{sp}^{2}$ ratio and surface oxygen functionalities in tetrahedral amorphous carbon (ta-C) thin film electrodes and implications of their electrochemical properties, J. Phys. Chem. C 120 (2016) 8298-8304. https://doi.org/10.1021/acs.jpcc. 6 b02342.

[19] N. Wester, J. Etula, T. Lilius, S. Sainio, T. Laurila, J. Koskinen, Selective detection of morphine in the presence of paracetamol with anodically pretreated dual layer Ti/tetrahedral amorphous carbon electrodes, Electrochem. Commun. 86 (2018) 166-170. https://doi.org/10.1016/j.elecom.2017.12.014.

[20] T. Laurila, V. Protopopova, S. Rhode, S. Sainio, T. Palomäki, M. Moram, J.M. Feliu, J. Koskinen, New electrochemically improved tetrahedral amorphous carbon films for biological applications, Diam. Relat. Mater. 49 (2014) 62-71. https://doi.org/10.1016/j.diamond.2014.08.007.

[21] T. Palomäki, S. Chumillas, S. Sainio, V. Protopopova, M. Kauppila, J. Koskinen, V. Climent, J.M. Feliu, T. Laurila, Electrochemical reactions of catechol, methylcatechol and dopamine at tetrahedral amorphous carbon (ta-C) thin film electrodes, Diam. Relat. Mater. 59 (2015) 30-39. https://doi.org/10.1016/j. diamond.2015.09.003.

[22] F. Ghorbani-Bidkorbeh, S. Shahrokhian, A. Mohammadi, R. Dinarvand, Simultaneous voltammetric determination of tramadol and acetaminophen using carbon nanoparticles modified glassy carbon electrode, Electrochim. Acta 55 (2010) 2752-2759. https://doi.org/10.1016/j.electacta.2009.12.052.

[23] A. Babaei, A.R. Taheri, M. Afrasiabi, A multi-walled carbon nanotube-modified glassy carbon electrode as a new sensor for the sensitive simultaneous determination of paracetamol and tramadol in pharmaceutical preparations and biological fluids, J. Braz. Chem. Soc. 22 (2011) 1549-1558. https://doi.org/ 10.1590/S0103-50532011000800020.

[24] B.J. Sanghavi, A.K. Srivastava, Simultaneous voltammetric determination of acetaminophen and tramadol using Dowex50wx2 and gold nanoparticles modified glassy carbon paste electrode, Anal. Chim. Acta 706 (2011) 246-254. https://doi.org/10.1016/j.aca.2011.08.040.

[25] A. Afkhami, H. Ghaedi, T. Madrakian, M. Ahmadi, H. Mahmood-Kashani, Fabrication of a new electrochemical sensor based on a new nano-molecularly imprinted polymer for highly selective and sensitive determination of tramadol in human urine samples, Biosens. Bioelectron. 44 (2013) 34-40. https://doi.org/10.1016/j.bios.2012.11.030.

[26] A. Afkhami, H. Khoshsafar, H. Bagheri, T. Madrakian, Preparation of NiFe2O4/ graphene nanocomposite and its application as a modifier for the fabrication of an electrochemical sensor for the simultaneous determination of tramadol and acetaminophen, Anal. Chim. Acta 831 (2014) 50-59. https://doi.org/10. 1016/j.aca.2014.04.061

[27] A.M. Santos, F.C. Vicentini, L.C.S. Figueiredo-Filho, P.B. Deroco, O. FatibelloFilho, Flow injection simultaneous determination of acetaminophen and tramadol in pharmaceutical and biological samples using multiple pulse amperometric detection with a boron-doped diamond electrode, Diam. Relat. Mater. 60 (2015) 1-8. https://doi.org/10.1016/j.diamond.2015.10.005.

[28] S. Chitravathi, N. Munichandraiah, Voltammetric determination of paracetamol, tramadol and caffeine using poly(Nile blue) modified glassy carbon electrode, J. Electroanal. Chem. 764 (2016) 93-103. https://doi.org/10.1016/j. jelechem.2016.01.021.

[29] D.G. Patil, N.M. Gokavi, A.M. Bagoji, S. Nandibewoor, Analytical \& electrochemical characterization and determination of tramadol drug using graphite pencil electrode, Anal. Bioanal. Chem. 8 (2016) 78-91.

[30] T. Madrakian, S. Alizadeh, M. Bahram, A. Afkhami, A novel electrochemical sensor based on magneto LDH/Fe3O4 nanoparticles@glassy carbon electrode for voltammetric determination of tramadol in real samples, Ionics (Kiel). 23 (2017) 1005-1015. https://doi.org/10.1016/j.msec.2015.07.054.

[31] M.A. Mohamed, S.A. Atty, N.N. Salama, C.E. Banks, Highly selective sensing platform utilizing graphene oxide and multiwalled carbon nanotubes for the sensitive determination of tramadol in the presence of Co-formulated drugs, Electroanalysis 29 (2017) 1038-1048. https://doi.org/10.1002/elan. 201600668.

[32] U.M. Stamer, F. Musshoff, M. Kobilay, B. Madea, A. Hoeft, F. Stuber, Concentrations of tramadol and O-desmethyltramadol enantiomers in different CYP2D6 genotypes, Clin. Pharmacol. Ther. 82 (2007) 41-47. https://doi.org/ 10.1038/sj.clpt.6100152.

[33] M. Szentirmay, C. Martin, Ion-exchange selectivity of Nafion films on electrode surfaces, Anal. Chem. 1902 (1984), 1898-1902, https://doi.org/10.1021/ ac00275a031.

[34] M.M. Davila, M.P. Elizalde, J. Mattusch, R. Wennrich, Study of the composite electrodes carbon - polyvinyl chloride and carbon - polyvinyl chloride/ Nafion by ex situ and in situ methods, Electrochim. Acta 46 (2001) 3189-3197.

[35] J. Chou, T.J. Ilgen, S. Gordon, A.D. Ranasinghe, E.W. McFarland, H. Metiu, S.K. Buratto, Investigation of the enhanced signals from cations and dopamine in electrochemical sensors coated with Nafion, J. Electroanal. Chem. 632 (2009) 97-101. https://doi.org/10.1016/j.jelechem.2009.04.002.

[36] J. Etula, N. Wester, S. Sainio, T. Laurila, J. Koskinen, Characterization and electrochemical properties of iron-doped tetrahedral amorphous carbon (taC) thin films, RSC Adv. 8 (2018) 26356-26363. https://doi.org/10.1039/ c8ra04719g.

[37] M. Soleimani, M.G. Afshar, A. Shafaat, G.A. Crespo, High-selective tramadol sensor based on modified molecularly imprinted polymer-carbon paste electrode with multiwalled carbon nanotubes, Electroanalysis 25 (2013) 1159-1168. https://doi.org/10.1002/elan.201200601. 